\title{
VARIABILIDADE ESPACIAL DE ATRIBUTOS QUÍMICOS DE UM ARGISSOLO PARA APLICAÇÃO DE INSUMOS À TAXA VARIÁVEL EM DIFERENTES FORMAS DE RELEVO ${ }^{1}$
}

\author{
DIOGO M. BARBIERI ${ }^{2}$, JOSÉ MARQUES JÚNIOR ${ }^{3}$, GENER T. PEREIRA ${ }^{4}$
}

RESUMO: A agricultura de precisão implica análise da variabilidade espacial de fatores de produção e a aplicação de insumos de forma localizada. Várias são as causas que condicionam a variabilidade espacial dos solos, sendo o relevo um dos fatores mais importantes. O presente estudo teve por objetivo analisar a variabilidade espacial dos atributos químicos do solo e a elaboração de mapas de necessidade de aplicação de insumos de forma localizada, em áreas com diferentes formas de relevo. Duas parcelas de 1 ha cada foram delimitadas em áreas com topografia côncava e convexa. Foram retiradas, em cada área, 242 amostras de solos em 121 pontos, nas profundidades de solo de 0,00-0,20 m e 0,20-0,40 m. Os resultados de análise química foram submetidos às análises da estatística descritiva, geoestatística e interpolação por krigagem. A área convexa apresentou maior variabilidade espacial do solo em relação a área côncava. A adoção da agricultura de precisão possibilitou economia de aproximadamente $25 \mathrm{~kg} \mathrm{ha}^{-1}$ de $\mathrm{P}_{2} \mathrm{O}_{5}$ na área côncava.

PALAVRAS-CHAVE: agricultura de precisão, geoestatística, manejo localizado.

\section{SPATIAL VARIABILITY OF CHEMICAL ATTRIBUTES IN AN ALFISOL FOR VARIABLE RATES OF INPUTS IN DIFFERENT FORMS OF RELIEF}

\begin{abstract}
The precision agriculture implies an analysis of spatial variability of production factors and the inputs application of located form. There are several factors that cause spatial variability in soils; relief is one of the most important ones. The objective of this study was to analyze the spatial variability, the chemical attributes of the soil and the elaboration of maps necessity for input application of located form, in areas with different relief forms. Two parcels of one hectare each were delimited in areas with concave and convex shaped topography. A set of 242 samples were collected from each area at 121 points in depths of $0.00-0.20 \mathrm{~m}$ and $0.20-0.40 \mathrm{~m}$. The data were submitted to the descriptive statistical analyses, geostatistics and interpolation for kriging. The convex area presented more spatial variability of the soil in relation the concave area. The adoption of precision agriculture made it possible to save approximately $25 \mathrm{~kg} \mathrm{ha}^{-1}$ of $\mathrm{P}_{2} \mathrm{O}_{5}$ in the concave area.
\end{abstract}

KEYWORDS: precision agriculture, geostatistics, specific management.

\section{INTRODUÇÃO}

A agricultura de precisão tem por objetivo aumentar a eficiência do manejo de técnicas agrícolas, sendo tecnologia em desenvolvimento que modifica técnicas existentes e incorpora novas tecnologias (BISCARO \& GARZELLA, 2006). Conforme ANTUNIASSI (1998) e BORGELT et al. (1994), o mapeamento detalhado dos fatores de produção e da aplicação localizada de insumos é o princípio básico do sistema. Dessa forma, a caracterização da variabilidade dos recursos é de fundamental importância para o uso efetivo da tecnologia de agricultura de precisão, tornando-se imprescindível para que essa técnica seja confiável (GREGO \& VIEIRA, 2005; SILVEIRA et al., 2000).

\footnotetext{
${ }^{1}$ Extraído da dissertação de mestrado do primeiro autor.

${ }^{2}$ Eng ${ }^{0}$ Agrônomo, Doutorando em Produção Vegetal, UNESP, Jaboticabal - SP, Fone: (0XX16) 3209.2672, diogombarbieri@yahoo.com.br

${ }^{3}$ Eng ${ }^{0}$ Agrônomo, Prof. Doutor, Departamento de Solos e Adubos, UNESP, Jaboticabal - SP.

${ }^{4}$ Estatístico, Prof. Dr., Departamento de Ciências Exatas, UNESP, Jaboticabal - SP.

Recebido pelo Conselho Editorial em: 4-9-2007

Aprovado pelo Conselho Editorial em: 10-10-2008
} 
Quando determinado atributo de solo varia de um local para outro, com algum grau de organização ou continuidade, expresso pela avaliação da dependência espacial, os resultados da estatística clássica são mais bem entendidos e aproveitados pela geoestatística (VIEIRA, 2000). Ajustado o semivariograma dos atributos, podem-se interpolar valores em qualquer posição no campo de estudo, sem tendência e com variância mínima. A esse método, dá-se o nome de krigagem (VIEIRA, 2000).

Em uma paisagem natural, o solo apresenta variabilidades espacial e temporal de seus atributos, resultantes da interação de processos que comandam os fatores responsáveis por sua formação (MONTEZANO et al., 2006). A associação da variabilidade espacial dos atributos de solos às formas da paisagem tem sido estudada por vários autores (MONTANARI et al., 2005; KRAVCHENKO et al., 2005; SOUZA et al., 2006a; SOUZA et al., 2006b) e tem contribuído para a identificação e o mapeamento de áreas mais homogêneas, com limites mais precisos entre elas (MARQUES JÚNIOR \& LEPSCH, 2000; FRANZEN et al., 2006; SOUZA et al., 2006b), o que permite que técnicas agronômicas possam ser transferidas com facilidade e economia para ambientes semelhantes.

A forma do relevo pode auxiliar na definição de esquemas amostrais, bem como no mapeamento de zonas de manejos físico e químico do solo (CARVALHO et al., 2003; SOUZA et al., 2006b). De acordo com MONTANARI et al. (2005) e FRANZEN et al. (2006), as pedoformas em áreas de mesma classe de solo e mesmo histórico de manejo de cana-de-açúcar, durante vários anos, influenciam na variabilidade dos atributos químicos dos solos de maneira sistemática. Estudos mostram que há maior variabilidade de atributos químicos e físicos em áreas de formas côncavas e convexas em relação às formas lineares, independentemente do histórico de manejo dessas áreas (SOUZA et al., 2004; SOUZA et al., 2006a).

A variabilidade espacial dos atributos químicos de solos possibilita a recomendação de doses de calcário, fósforo e potássio com taxas variadas, proporcionando economia e maior eficiência na aplicação (CHANG et al., 2003; WANG et al., 2006), onde os limites de áreas mais homogêneas podem ser definidos pelas características do relevo (MARQUES JÚNIOR \& LEPSCH, 2000; FRANZEN et al., 2002). Desse modo, o objetivo deste trabalho foi analisar a variabilidade espacial dos atributos químicos do solo e a elaboração de mapas de necessidade de aplicação de insumos de forma localizada, em áreas com diferentes formas de relevo.

\section{MATERIAL E MÉTODOS}

A área de estudo está localizada na região de Catanduva - SP, inserida na província geomorfológica do Planalto Ocidental Paulista. O clima da região foi classificado, pelo método de Köeppen, como tropical quente úmido, tipo Aw, seco no inverno, com precipitação média de $1.350 \mathrm{~mm}$, temperatura média anual de $23{ }^{\circ} \mathrm{C}$, com temperatura no mês mais quente superior a $22{ }^{\circ} \mathrm{C}$ e a do mês mais frio inferior a $18{ }^{\circ} \mathrm{C}$, e umidade relativa do ar de $74 \%$. A vegetação primária da região de Catanduva foi classificada como floresta pluvial estacional de Cerrado, sendo a área utilizada há mais de 20 anos com o cultivo de cana-de-açúcar (SANCHEZ, 2003). As coordenadas geográficas da área são: latitude $21^{\circ} 05^{\prime} \mathrm{S}$ e longitude $49^{\circ} 01^{\prime} \mathrm{W}$. O material de origem dos solos foi mapeado como rocha arenítica do Grupo Bauru, Formação Adamantina (IPT, 1981). O solo da área de estudo foi classificado como Argissolo Vermelho-Amarelo eutrófico, textura média/argilosa (EMBRAPA, 1999).

Para o georreferenciamento da área, foi utilizado um receptor GPS TRIMBLE, modelo 4.600 LS Geodésico, com precisão $(0,5 \mathrm{~cm}+1 \mathrm{ppm})$. Elaborou-se o modelo digital de elevação que, juntamente com as atividades de campo, possibilitou a identificação e a separação das formas do terreno e sua posterior classificação geomorfológica, conforme TROEH (1965). De acordo com essa classificação, em uma área de 200 ha, foram mapeadas duas curvaturas, sendo uma côncava e outra convexa (Figura 1).

Nessa área, duas parcelas de 1 ha foram delimitadas, sendo uma parcela localizada na face 
côncava e a outra na face convexa. Uma malha de espaçamento de 10 x $10 \mathrm{~m}$ foi confeccionada em cada uma das parcelas, e os pontos localizados nos cruzamentos das malhas foram georreferenciados. Os solos foram amostrados em setembro de 2005, nos pontos de cruzamento da malha, nas profundidades de 0,0-0,2 m; 0,2-0,4 m, perfazendo o total de 121 pontos (242 amostras simples) em cada malha (Figura 1), sendo as amostras levadas ao laboratório e submetidas à análise química. A acidez potencial $(\mathrm{H}+\mathrm{Al})$ foi obtida segundo RAIJ et al. (2001). O cálcio $(\mathrm{Ca})$, o magnésio $(\mathrm{Mg})$, o potássio $(\mathrm{K})$, o fósforo disponível $(\mathrm{P})$ e a matéria orgânica foram extraídos utilizando o método da resina trocadora de íons proposta por RAIJ et al. (2001). Com base nos resultados das análises químicas, foram calculadas a soma de bases (SB) e a capacidade de troca catiônica (CTC) para posterior cálculo da saturação por bases (V\%).

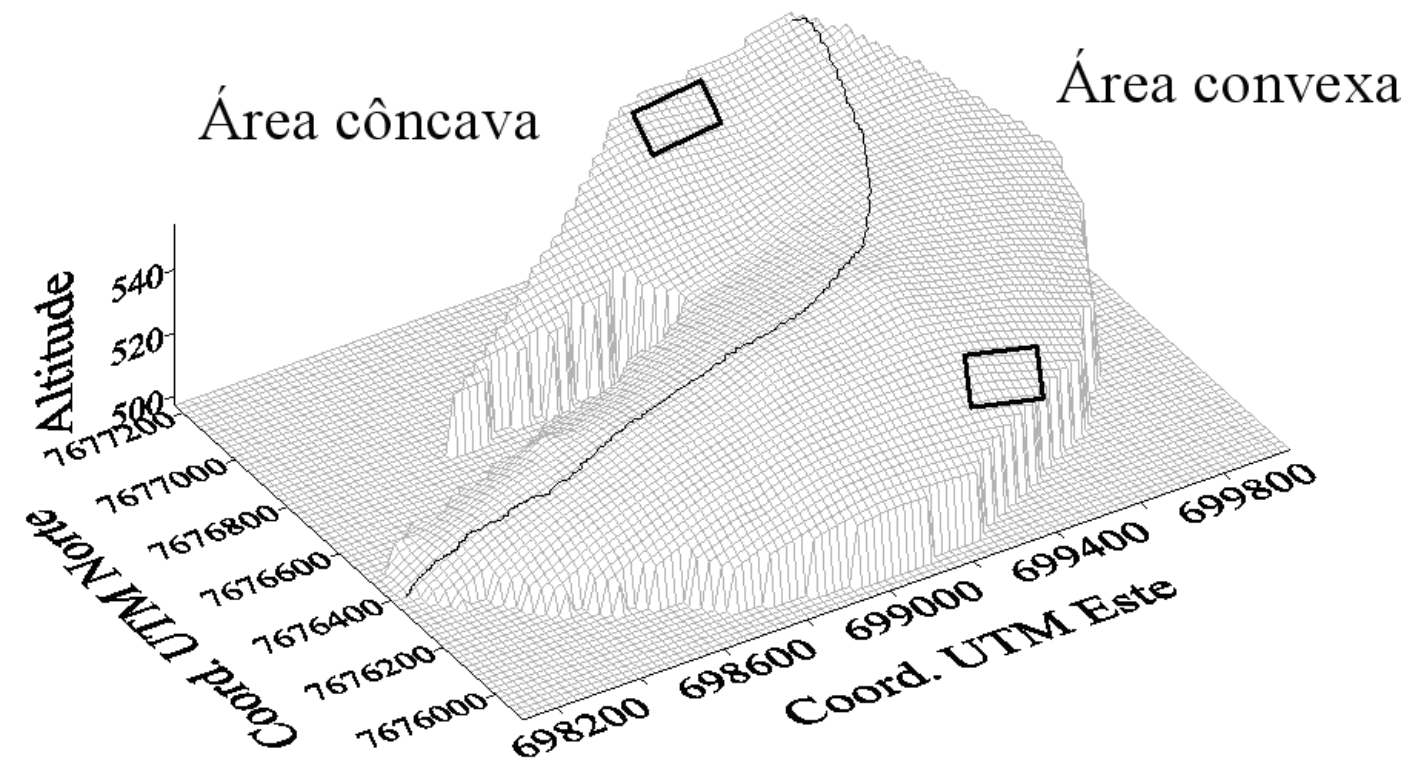

FIGURA 1. Modelo digital de elevação do terreno e localização das duas áreas de amostragem nas faces côncava e convexa (modificada de SANCHEZ, 2003). Digital model of rise and localization of areas concave and convex (modified figure of SANCHEZ, 2003).

O histórico de manejo das duas áreas é idêntico e a variedade de cana-de-açúcar plantada em março de 2003 foi a SP801842. A adubação líquida realizada antes do plantio foi de $870 \mathrm{~kg} \mathrm{ha}^{-1} \mathrm{da}$ fórmula 04-12-10 ( $\left.\mathrm{N}-\mathrm{P}_{2} \mathrm{O}_{5}-\mathrm{K}_{2} \mathrm{O}\right)$, e na adubação de cana-soca realizada após o primeiro corte (2004), foram utilizados $620 \mathrm{~kg} \mathrm{ha}^{-1}$ da fórmula 10-00-10 $\left(\mathrm{N}-\mathrm{P}_{2} \mathrm{O}_{5}-\mathrm{K}_{2} \mathrm{O}\right)$. Torta de filtro e vinhaça não foram aplicadas nas áreas de amostragem.

A necessidade de calagem e adubação com fósforo e potássio foi estimada de duas maneiras: uma, considerando-se a média dos 121 pontos, e a outra, considerando-se a variabilidade espacial dos atributos nas malhas. Para o cálculo da necessidade de calcário, utilizou-se da fórmula proposta por RAIJ et al. (1997), que leva em consideração a saturação por bases atual do solo, a CTC e o nível de saturação por bases ideal da cultura que, no caso da cana-de-açúcar no Estado de São Paulo, é de $60 \%$, considerando o PRNT de $100 \%$. Para o cálculo da necessidade de adubação com fósforo e potássio, foram utilizadas as tabelas de adubação propostas por RAIJ et al. (1997), sendo a produtividade esperada considerada de 100 a $150 \mathrm{t} \mathrm{ha}^{-1}$.

Os resultados obtidos foram submetidos à análise estatística descritiva, obtendo-se média, mediana, coeficiente de variação, assimetria e curtose e tipo de distribuição dos dados com o uso do pacote estatístico SAS (SCHLOTZHAVER \& LITTELL, 1997). Posteriormente, foram realizadas análises geoestatísticas por meio da construção de semivariogramas com o auxílio do programa $\mathrm{GS}^{+}$ (ROBERTSON, 1998) e confecção dos mapas de krigagem utilizando-se do programa SURFER (1999). 


\section{RESULTADOS E DISCUSSÃO}

Os teores de $\mathrm{P}, \mathrm{K}$ e V\%, bem como as necessidades médias de aplicação de nutrientes e calcário são apresentados na Tabela 1. Registrou-se, no solo da área convexa, necessidade de maiores quantidades de fósforo e de calcário, comparada com a área côncava. Porém, analisando a necessidade média de aplicação de potássio, nota-se que as duas áreas devem receber a mesma quantidade desse nutriente (Tabela 1). Os valores de máximo e mínimo, para os atributos fósforo, potássio e V\%, nas duas profundidades estudadas, apresentaram variação dentro das classes de fertilidade de solos propostas por RAIJ et al. (1997) para a cultura da cana-de-açúcar.

De acordo com a classificação de WARRICK \& NIELSEN (1980), apenas a saturação por bases na área côncava, na profundidade de 0,00-0,20 m, apresentou coeficiente de variação baixo. $\mathrm{O}$ restante dos atributos apresentou coeficientes de variação médio ou alto nas duas profundidades estudadas. Comparando-se os coeficientes de variação obtidos entre as duas áreas estudadas, observa-se que os valores encontrados na área convexa são maiores do que os da área côncava, com exceção do potássio e da necessidade de fósforo na profundidade de 0,00-0,20 m, refletindo maior variação dos dados na área convexa (Tabela 1), o que pode ser confirmado pelos diferentes comportamentos espaciais desses atributos (Tabela 2).

Os resultados referentes ao teste Kolmogorov-Smirnov indicaram normalidade para a maioria dos atributos. O teor e a necessidade de potássio na profundidade de 0,00-0,20 m, nas duas áreas estudadas, e a necessidade de fósforo na área convexa não apresentaram distribuição normal dos dados. Na profundidade de 0,20-0,40 m, apenas o fósforo, na área côncava, e o potássio, na área convexa, não apresentaram normalidade na distribuição dos dados. MONTEZANO et al. (2006) e MONTANARI et al. (2005), estudando variabilidade de atributos químicos, não encontraram distribuição normal para a maioria dos atributos estudados.

TABELA 1. Resultados da estatística descritiva para os atributos químicos na profundidade de solo de $0,00-0,20 \mathrm{~m}$ e $0,20-0,40 \mathrm{~m}$. Results of the descriptive statistics of chemical attributes at $0.00-0.20 \mathrm{~m}$ and $0.20-0.40 \mathrm{~m}$ soil depths.

\begin{tabular}{|c|c|c|c|c|c|c|c|c|c|}
\hline \multirow{2}{*}{ Parâmetros } & \multirow{2}{*}{ Área } & \multirow{2}{*}{ Média } & \multirow{2}{*}{ Mediana } & \multirow{2}{*}{ Mín. } & \multirow{2}{*}{ Máx. } & \multirow{2}{*}{$\mathrm{p}^{1}$} & \multicolumn{3}{|c|}{ Coeficientes } \\
\hline & & & & & & & Var. (\%) & Assimetria & Curtose \\
\hline \multicolumn{10}{|c|}{$0,00-0,20 \mathrm{~m}$} \\
\hline \multirow{2}{*}{$\mathrm{P}\left(\mathrm{MG} \mathrm{dm}^{-3}\right)$} & Côncava & $12 \mathrm{a}$ & 11 & 4 & 25 & 0,07 & 41 & 0,64 & $-0,23$ \\
\hline & Convexa & $7 \mathrm{~b}$ & 7 & 1 & 14 & 0,15 & 51 & 0,01 & $-0,54$ \\
\hline \multirow{2}{*}{$\mathrm{K}\left(\mathrm{mmolc} \mathrm{dm}^{-3}\right)$} & Côncava & $1,3 \mathrm{a}$ & 1,3 & 0,7 & 2,4 & 0,04 & 29 & 0,64 & $-0,23$ \\
\hline & Convexa & $1,4 \mathrm{a}$ & 1,4 & 0,8 & 2,5 & 0,02 & 28 & 0,75 & $-0,10$ \\
\hline \multirow{2}{*}{$\mathrm{V} \%$} & Côncava & $71 \mathrm{a}$ & 71 & 49 & 88 & 0,15 & 11 & $-0,33$ & $-0,08$ \\
\hline & Convexa & $50 \mathrm{~b}$ & 51 & 19 & 73 & 0,15 & 25 & $-0,23$ & $-0,53$ \\
\hline \multirow{2}{*}{ 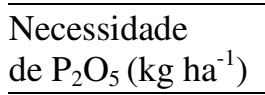 } & Côncava & $121 \mathrm{a}$ & 120 & 80 & 180 & 0,15 & 24 & 0,68 & 0,31 \\
\hline & Convexa & $150 \mathrm{~b}$ & 120 & 120 & 180 & 0,01 & 20 & 0,28 & $-1,95$ \\
\hline \multirow{2}{*}{$\begin{array}{l}\text { Necessidade } \\
\text { de } \mathrm{K}_{2} \mathrm{O}\left(\mathrm{kg} \mathrm{ha}^{-1}\right)\end{array}$} & Côncava & $110 \mathrm{a}$ & 120 & 80 & 150 & 0,01 & 16 & $-0,96$ & $-0,74$ \\
\hline & Convexa & $110 \mathrm{a}$ & 120 & 80 & 120 & 0,01 & 18 & $-0,57$ & $-1,69$ \\
\hline \multirow{2}{*}{$\begin{array}{l}\text { Necessidade de } \\
\text { Calcário }\left(\mathrm{t} \mathrm{ha}^{-1}\right)\end{array}$} & Côncava & -- & -- & -- & -- & -- & -- & -- & -- \\
\hline & Convexa & 1,0 & 0,6 & 0 & 2,5 & 0,15 & 56 & 0,7 & $-0,38$ \\
\hline \multicolumn{10}{|c|}{$0,20-0,40 \mathrm{~m}$} \\
\hline \multirow{2}{*}{$\mathrm{P}\left(\mathrm{MG} \mathrm{dm}^{-3}\right)$} & Côncava & $11 \mathrm{a}$ & 10 & 3 & 24 & 0,03 & 46 & 0,69 & $-0,1$ \\
\hline & Convexa & $5,9 \mathrm{~b}$ & 6,0 & 1 & 15 & 0,15 & 54 & 0,20 & $-0,48$ \\
\hline \multirow{2}{*}{$\mathrm{K}\left(\mathrm{mmolc} \mathrm{dm}^{-3}\right)$} & Côncava & $1,1 \mathrm{a}$ & 1,1 & 0,7 & 1,8 & 0,07 & 24 & 0,60 & $-0,41$ \\
\hline & Convexa & $1,2 \mathrm{a}$ & 1,2 & 0,8 & 2,0 & 0,01 & 25 & 0,94 & $-0,48$ \\
\hline \multirow{2}{*}{$\mathrm{V} \%$} & Côncava & $67 a$ & 69 & 38 & 91 & 0,15 & 14 & $-0,28$ & 0,06 \\
\hline & Convexa & $42 b$ & 43 & 18 & 68 & 0,15 & 28 & 0,20 & $-0,48$ \\
\hline
\end{tabular}


No presente estudo, foram encontrados valores de assimetria e de curtose próximos a zero para todos os atributos estudados (Tabela 1). A utilização de técnicas geoestatísticas exige apenas simetria da distribuição dos dados (VIEIRA, 2000). Outra indicação de simetria da distribuição dos dados são os valores de média e de mediana próximos, com exceção da necessidade de fósforo na área convexa e da necessidade de potássio nas duas áreas (Tabela 1). Resultados semelhantes para atributos de solos foram encontrados por MONTANARI et al. (2005) e SOUZA et al. (2006a). Isso demonstra que todos os atributos envolvidos no estudo estão se aproximando de distribuição normal, indicando que os dados estão adequados para o uso da geoestatística.

TABELA 2. Resultado da análise geoestatística dos semivariogramas nas profundidades de solo de $0,00-0,20 \mathrm{~m}$ e $0,20-0,40 \mathrm{~m}$. Result of the geostatistics analysis of the semivariograms at $0.00-0.20 \mathrm{~m}$ and $0.20-0.40 \mathrm{~m}$ soil depths.

\begin{tabular}{|c|c|c|c|c|c|c|c|}
\hline Parâmetros & Área & Modelo & $\begin{array}{c}\mathrm{a} \\
(\mathrm{m})\end{array}$ & Co & $\mathrm{C}_{0}+\mathrm{C}_{1}$ & $\mathrm{Co} /\left(\mathrm{Co}+\mathrm{C}_{1}\right) \%$ & $\mathrm{r}^{2}$ \\
\hline \multicolumn{8}{|c|}{$0,00-0,20 \mathrm{~m}$} \\
\hline \multirow{2}{*}{$\mathrm{P}\left(\mathrm{mg} \mathrm{dm}^{-3}\right)$} & Côncava & Esférico & 101 & 11,57 & 23,49 & 49 & 0,9 \\
\hline & Convexa & Esférico & 26 & 0,01 & 11,4 & 0,1 & 0,8 \\
\hline \multirow{2}{*}{$\mathrm{K}\left(\mathrm{mmol}_{\mathrm{c}} \mathrm{dm}^{-3}\right)$} & Côncava & Exponencial & 37 & 0,013 & 0,130 & 10 & 0,9 \\
\hline & Convexa & Esférico & 23 & 0,002 & 0,12 & 2 & 0,8 \\
\hline \multirow{2}{*}{ V \% } & Côncava & Esférico & 35 & 0,8 & 45,18 & 2 & 0,9 \\
\hline & Convexa & Exponencial & 25 & 24,3 & 128 & 19 & 0,8 \\
\hline \multirow{2}{*}{$\begin{array}{l}\text { Necessidade de } \mathrm{P}_{2} \mathrm{O}_{5} \\
\left(\mathrm{~kg} \mathrm{ha}^{-1}\right)\end{array}$} & Côncava & Gaussiano & 42 & 414 & 828 & 50 & 0,9 \\
\hline & Convexa & Esférico & 25 & 1 & 901 & 0,1 & 0,9 \\
\hline \multirow{2}{*}{$\begin{array}{l}\text { Necessidade de } \mathrm{K}_{2} \mathrm{O} \\
\left(\mathrm{kg} \mathrm{ha}^{-1}\right)\end{array}$} & Côncava & Esférico & 21 & 3,1 & 313 & 1 & 0,5 \\
\hline & Convexa & Esférico & 18 & 11 & 380 & 3 & 0,9 \\
\hline Necessidade de & Côncava & - & - & - & - & - & - \\
\hline Calcário $\left(\mathrm{t} \mathrm{ha}^{-1}\right)$ & Convexa & Esférico & 22 & 0,001 & 0,58 & 0,2 & 0,7 \\
\hline \multicolumn{8}{|c|}{$0,20-0,40 \mathrm{~m}$} \\
\hline \multirow{2}{*}{$\mathrm{P}\left(\mathrm{mg} \mathrm{dm}^{-3}\right)$} & Côncava & Esférico & 89 & 9,2 & 23,21 & 40 & 0,9 \\
\hline & Convexa & Esférico & 23 & 0,4 & 10,01 & 4 & 0,8 \\
\hline \multirow{2}{*}{$\mathrm{K}\left(\mathrm{mmol}_{\mathrm{c}} \mathrm{dm}^{-3}\right)$} & Côncava & Esférico & 84 & 0,033 & 0,082 & 40 & 0,9 \\
\hline & Convexa & Esférico & 22 & 0,006 & 0,153 & 4 & 0,8 \\
\hline \multirow{2}{*}{$\mathrm{V} \%$} & Côncava & Exponencial & 132 & 45,8 & 103,9 & 44 & 0,9 \\
\hline & Convexa & Gaussiano & 100 & 89,9 & 179,9 & 50 & 0,9 \\
\hline
\end{tabular}

a - alcance; $\mathrm{C}_{\mathrm{o}}$ - efeito pepita; $\mathrm{C}_{0}+\mathrm{C}_{1}$ - patamar; $\mathrm{Co} /\left(\mathrm{Co}+\mathrm{C}_{1}\right) \%$ - grau de dependência espacial

Todos os atributos avaliados apresentaram dependência espacial, a qual é expressa por meio dos ajustes aos modelos de semivariogramas (Tabela 2 e Figura 2). Os dados da maioria dos atributos estudados ajustaram-se ao modelo matemático esférico; este, segundo GREGO \& VIEIRA (2005), é o modelo que predomina para os atributos estudados em Ciência do Solo.

Conforme classificação de CAMBARDELLA et al. (1994), o atributo fósforo, nas duas profundidades de solo, necessidade de fósforo, na profundidade de 0,00-0,20 m, e o teor de potássio, na profundidade de 0,20-0,40 m, apresentaram grau de dependência espacial médio para a área côncava. Já a saturação por bases apresentou médio grau de dependência espacial nas duas áreas analisadas, na profundidade de 0,20-0,40 m. O restante dos atributos apresentou forte grau de dependência espacial.

O comportamento espacial diferenciado entre os atributos também pode ser observado pelo alcance (Tabela 2), que registrou seus maiores valores para área côncava. Os menores valores de alcance, encontrados na área convexa, confirmam a maior variabilidade espacial dos atributos químicos existentes nessa área, reflexo da influência do relevo no comportamento espacial desses atributos (Tabela 2 e Figura 2). Os resultados do comportamento espacial dos atributos na profundidade de 0,20-0,40 m (Tabela 2) concordam com os da camada superficial, confirmando o 
caráter pedogeomórfico como causa da variabilidade dos atributos do solo (CARVALHO et al., 2003; MONTEZANO et al., 2006).

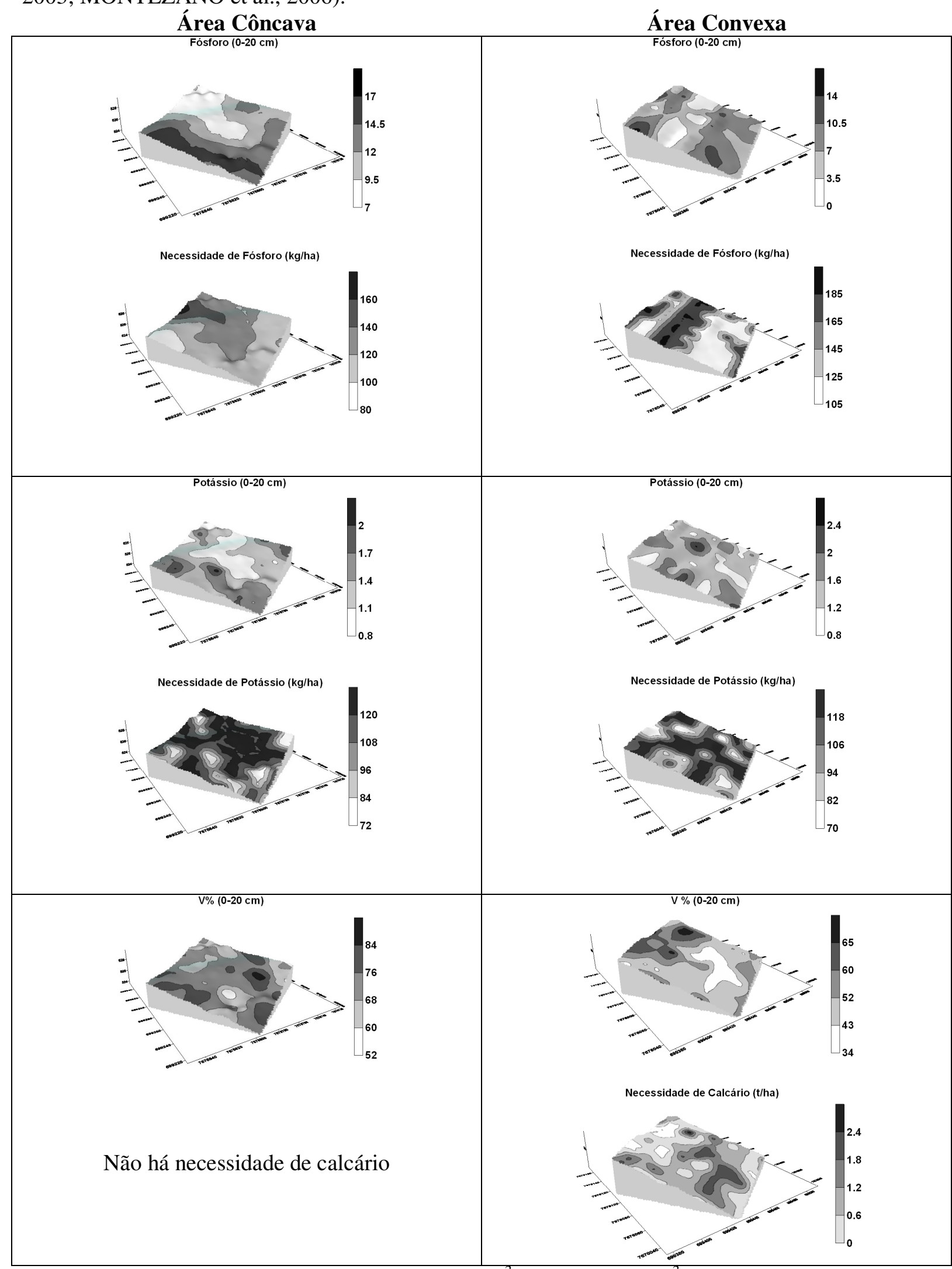

FIGURA 2. Mapas de krigagem para o $\mathrm{P}\left(\mathrm{mg} \mathrm{dm}^{-3}\right), \mathrm{K}\left(\mathrm{mmol}_{\mathrm{c}} \mathrm{dm}^{-3}\right), \mathrm{V}(\%)$ e necessidades de aplicação. Kriging maps for the $\mathrm{P}\left(\mathrm{mg} \mathrm{dm}^{-3}\right), \mathrm{K}\left(\mathrm{mmol}_{\mathbf{c}} \mathrm{dm}^{-3}\right), \mathrm{V}(\%)$ and necessities of application. 
De acordo com MARQUES JÚNIOR \& LEPSCH (2000) e KRAVCHENKO \& BULLOCK (2000), para o entendimento das causas da variabilidade do solo, é preciso conhecer os processos do solo que operam em locais específicos. Esses processos estão muito ligados à água, sendo o relevo o principal controlador da intensidade e do fluxo de água em uma determinada área. Nesse sentido, a compartimentação das formas do relevo revela ser eficiente para a identificação e o mapeamento de áreas com variabilidade controlada, bem como a transferência de informações (FRANZEN et al., 2006).

As doses recomendadas para a aplicação de fósforo na área côncava variaram de 80 a $160 \mathrm{~kg} \mathrm{ha}^{-1}$ quando levados em conta os valores de cada ponto amostrado da área (Figura 2). Considerando a área homogênea, como feito na agricultura tradicional, a dose aplicada na área seria de $120 \mathrm{~kg} \mathrm{ha}^{-1}$ de $\mathrm{P}_{2} \mathrm{O}_{5}$ (Tabela 1). Dessa forma, pela adubação baseada na média, boa parte da área deverá receber quantidade de adubo fosfatado superior ou inferior à dose realmente necessária (CHANG et al., 2003; WANG et al., 2006; BISCARO \& GARZELLA, 2006).

Para o fósforo, na área convexa, as doses a serem aplicadas variaram de 105 a $185 \mathrm{~kg} \mathrm{ha}^{-1}$ (Figura 2), e a aplicação média é de $150 \mathrm{~kg} \mathrm{ha}^{-1}$ (Tabela 1). O mapa da necessidade de fósforo

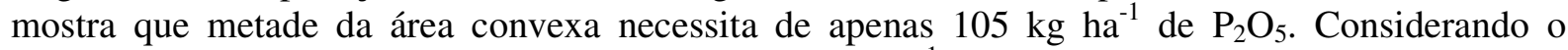
tamanho da área de 1 ha e a aplicação média de $150 \mathrm{~kg} \mathrm{ha}^{-1}$, a adoção das técnicas de agricultura de precisão gerariam economia de aproximadamnte $25 \mathrm{~kg} \mathrm{ha}^{-1}$ de $\mathrm{P}_{2} \mathrm{O}_{5}$, uma vez que metade da área receberia apenas $105 \mathrm{~kg} \mathrm{ha}^{-1}$ do fertilizante. Dessa forma, a utilização da técnica levaria a maiores eficiência e economia do fertilizante, proporcionando, assim, além de menores custos na adubação, menor impacto ambiental por evitar excesso de fósforo na área (CHANG et al., 2003; BISCARO \& GARZELLA, 2006).

Para os mapas de necessidade de potássio, os valores variaram de 70 a $120 \mathrm{~kg} \mathrm{ha}^{-1}$ para ambas as áreas estudadas (Figura 2). O valor médio da necessidade de aplicação de potássio nas duas áreas é de $110 \mathrm{~kg} \mathrm{ha}^{-1}$ (Tabela 1). Entretanto, observa-se que há vários locais que necessitam de apenas 70 ; 80 ou $90 \mathrm{~kg} \mathrm{ha}^{-1}$ e outros de $120 \mathrm{~kg} \mathrm{ha}^{-1}$. Desse modo, também para a adubação potássica realizada pela média dos pontos, os locais receberiam menor dose que a necessária, enquanto em outros locais a quantidade de adubo aplicado seria maior que a dose ótima.

A necessidade de calcário informada pelos mapas obtidos por interpolação (Figura 2) variou de zero a 2,4 $\mathrm{t} \mathrm{ha}^{-1}$ na área convexa; na área côncava, não seria recomendado realizar a calagem. Para a área convexa, o cálculo da necessidade de calcário, utilizando-se do método convencional sem considerar a variabilidade espacial de atributos da acidez do solo, indicou ser necessário aplicar 1,0 t ha ${ }^{-1}$ de calcário (Tabela 1). Assim, boa parte da área receberia calcário de forma desnecessária, onerando os custos de aplicação e causando inadequação ao equilíbrio na absorção de íons pelas plantas.

\section{CONCLUSÕES}

A área convexa apresentou maior variabilidade espacial dos atributos químicos, comparado com o solo da área côncava. Os limites das formas do relevo demonstraram ser indicadores das zonas específicas para a aplicação de insumos a taxas variadas. Mapas para aplicação de insumos à taxa variada, elaborados por técnicas geoestatísticas, indicam maior eficiência para a aplicação de calcário, fósforo e potássio.

\section{AGRADECIMENTOS}

À Usina São Domingos, pela concessão da área e pelo apoio na realização do trabalho.

\section{REFERÊNCIAS}

ANTUNIASSI, U. R. Agricultura de precisão: aplicação localizada de agrotóxicos. In: GUEDES, J.V.C.; DORNELES, S.H.B. (Org.). Tecnologia e segurança na aplicação dos agrotóxicos - novas 
tecnologias. Santa Maria: Departamento de Defesa Fitossanitária; Sociedade de Agronomia de Santa Maria, 1998. p.53-63.

BISCARO, A.S.; GARZELLA, T.C. Agricultura de precisão: uma valiosa ferramenta para redução de contaminação ambiental decorrente do uso de fertilizantes minerais nitrogenados. In: CONGRESSO BRASILEIRO DE AGRICULTURA DE PRECISÃO, 2., 2006, São Pedro. Anais... Piracicaba: ESALQ, 2006. 1 CD-ROM.

BORGELT, S.C.; SEARCY, S.W.; STOUT, B.A.; MULLA, D.J.J. Spatially variable liming rates: a method for determination. Transactions of the ASAE, St. Joseph, v.37, n.5, p.1.499-1.507, 1994.

CAMBARDELLA, C.A.; MOORMAN, T.B.; NOVAK, J.M.; PARKIN, T.B.; KARLEN, D.L.; TURCO, R.F.; KONOPKA, A. E. Field-scale variability of soil properties in Central Iowa. Soil Science Society of American Journal, Madison, v.58, n.5, p.1501-11, 1994.

CARVALHO, M.P.; TAKEDA, E.Y. ; FREDDI, O.S. Variabilidade espacial de atributos de um solo sob videira em Vitória Brasil (SP). Revista Brasileira de Ciência do Solo, Viçosa, v.27, n.6, p.695-703, 2003.

CHANG, J.; CLAY, D.E.; CARLSON, C.G.; CLAY, S.A.; MALO, D.D.; BERG, R.; KLEINJAN, J.; WIEBOLD, W. Different techniques to identify management zones impact nitrogen and phosphorus sampling variability. Agronomy Journal, Madison, v.95, p.1.550-9, 2003.

EMBRAPA. EMPRESA BRASILEIRA DE PESQUISA AGROPECUÁRIA. Centro Nacional de Pesquisa de Solos. Sistema Brasileiro de Classificação de Solos. Brasília, 1999. 412 p.

FRANZEN, D.W.; HOPKINS, D.H.; SWEENEY, M.D.; ULMER, M.K.; HALVORSON, A.D. Evaluation of soil survey scale for zone development of site-specific-nitrogen management. Agronomy Journal, Madison, v.94, p.381-9, 2002.

FRANZEN, D.W.; NANNA, T.; NORVELL, W.A. A survey of soil attributes in North Dakota by landscape position. Agronomy Journal, Madison, v.98, p.1.015-22, 2006.

GREGO, C.R.; VIEIRA, S.R. Variabilidade espacial de propriedades físicas do solo em uma parcela experimental. Revista Brasileira de Ciência do Solo, Viçosa, v.29, n.2, p.169-77, 2005.

IPT. INSTITUTO DE PESQUISAS TECNOLÓGICAS DO ESTADO DE SÃO PAULO. Mapa geomorfológico do Estado de São Paulo. Escala - 1:1.000.000. São Paulo, 1981, v.1. 94 p.

KRAVCHENKO, A.N.; BULLOCK, D.G. Correlation of corn and soybean grainyield with topography and soil properties. Agronomy Journal, Madison, v.92, p.75-83, 2000.

KRAVCHENKO, A.N.; ROBERTSON, G.P.; THELEN, K.D.; HARWOOD, R.R.. Management, topographical, and weather effects on spatial variability of crop grain yields. Agronomy Journal, Madison, v.97, p.514-23, 2005.

MARQUES JÚNIOR, J.; LEPSCH, I.F. Depósitos superficiais neocenozoicos, superfícies geomórficas e solos em Monte Alto - SP. Geociência, São Paulo, v.19, n.1, p.90-106, 2000.

MONTANARI, R.; MARQUES JÚNIOR, J.; PEREIRA G. T.; SOUZA, Z. M. Forma da paisagem como critério para otimização amostral de Latossolos sob cultivo de cana-de-açúcar. Pesquisa Agropecuária Brasileira, Brasília, v.40, n.1, p.69-77, 2005.

MONTEZANO, Z.F.; CORAZZA, E.J.; MURAOKA, T. Variabilidade espacial da fertilidade do solo em área cultivada e manejada homogeneamente. Revista Brasileira de Ciência do Solo, Viçosa, v.30, n.5, p.839-47, 2006.

RAIJ, B. van; ANDRADE, J.C.; CANTARELLA, H.; QUAGGIO, J.A. (Ed.) Análise química para avaliação da fertilidade de solos tropicais. Campinas: Instituto Agronômico, 2001. 285 p. 
RAIJ, B. van; CANTARELLA, H.; QUAGGIO, J.A.; FURLANI, A.M.C. Recomendações de adubação e calagem para o estado de São Paulo. 2.ed. Campinas: Instituto Agronômico/Fundação IAC, 1997. 285 p. (Boletim Técnico, 100).

ROBERTSON, G.P. $G S^{+}$: Geostatistics for the environmental sciences - GS ${ }^{+}$User's Guide. Plainwell: Gamma Design Software, 1998. 152 p.

SANCHEZ, R.S. Zonas de manejo caracterizadas por meio de pedoformas em áreas sob o cultivo de cana-de-açúcar. 2003. 92 f. Dissertação (Mestrado em Ciência do Solo) - Faculdade de Ciências Agrárias e Veterinárias, Universidade Estadual Paulista, Jaboticabal, 2003.

SCHLOTZHAVER, S.D.; LITTELL, R.C. SAS: system for elementary statistical analysis. $2^{\text {nd }}$ ed. Cary, 1997. 905 p.

SILVEIRA, P.M.; ZIMMERMANN, F.J.P.; SILVA, S.C.; CUNHA, A.A. Amostragem e variabilidade espacial de características químicas de um Latossolo submetido a diferentes sistemas de preparo. Pesquisa Agropecuária Brasileira, Brasília, v.35, n.10, p. 2.057-64, 2000.

SOUZA, Z.M.; MARQUES JÚNIOR, J.; PEREIRA, G.T.; MOREIRA, L.F. Variabilidade espacial do $\mathrm{pH}, \mathrm{Ca}, \mathrm{Mg}$, e V\% do solo em diferentes formas do relevo sob cultivo de cana-de-açúcar. Ciência Rural, Santa Maria, v.34, n.6, p.1.763-71, 2004.

SOUZA, Z.M.; MARQUES JÚNIOR, J.; PEREIRA, G.T.; BARBIERI, D.M. Effects of relief shapes on soil chemical properties of a Oxisol cultivated with sugar cane. Scientia Agrícola, Piracicaba, v.63, n. 2 p.161-8, 2006a.

SOUZA, Z.M.; MARQUES JÚNIOR, J.; PEREIRA G.T.; MONTANARI, R. Otimização amostral de atributos de Latossolos considerando aspectos solo-relevo. Ciência Rural, Santa Maria, v.36, n.3, p.829-36, 2006b.

SURFER for Windows. Realese 7.0. Contouring and 3D surface mapping for scientist's engineers. User's guide. New York: Golden Software, 1999. 619 p.

TROEH, F.R. Landform equations fitted to contour maps. American Journal of Science, New Haven, v.263, n.3, p.616-27, 1965.

VIEIRA, S.R. Geoestatística em estudos de variabilidade espacial do solo. In: NOVAIS, R.F.; ALVARES, V.H.; SCHAEFER, C.E.G.R. Tópicos em Ciência do Solo. Viçosa: Sociedade Brasileira de Ciência do Solo, 2000. p.1-54.

WANG B.H.; JIN, L.; WANG, B. Improvement of soil nutrient management via information technology. Better Crops, Norcross, v.90, n.3, p.30-2, 2006.

WARRICK, A.W.; NIELSEN, D.R. Spatial variability of soil physical properties in the field. In: HILLEL, D.(Ed.). Applications of Soil Physics. New York: Academic Press, 1980. cap. 2, p.319-44. 